\title{
ATLAS LAr Calorimeter Performance and LHC Run-2 Commissioning
}

Fabian Spettel ${ }^{\mathrm{a}}$, on behalf of the ATLAS collaboration

${ }^{a}$ Max-Planck-Institut für Physik, München, Germany

\begin{abstract}
The ATLAS detector was built to study proton-proton collisions produced by the Large Hadron Collider (LHC) at a center of mass energy of up to $14 \mathrm{TeV}$. The Liquid Argon (LAr) calorimeters are used for all electromagnetic calorimetry as well as the hadronic calorimetry in the endcap and forward regions. They have shown excellent performance during the first LHC data taking campaign, from 2010 to 2012, so-called Run 1, at a peak luminosity of $8 \times 10^{33} \mathrm{~cm}^{-2} \mathrm{~s}^{-1}$. During the next run, peak luminosities of $1.5 \times 10^{34} \mathrm{~cm}^{-2} \mathrm{~s}^{-1}$ and even higher are expected at a $25 \mathrm{~ns}$ bunch spacing. Such a high collision rate may have an impact on the quality of the energy reconstruction which is attempted to be maintained at a high level using a calibration procedure described in this contribution. It also poses major challenges to the first level of the trigger system which is constrained to a maximal rate of 100 $\mathrm{kHz}$. For Run-3, scheduled to start in 2019, instantaneous luminosity as high as $3 \times 10^{34} \mathrm{~cm}^{-2} \mathrm{~s}^{-1}$ are foreseen imposing an upgrade of the LAr trigger system to maintain its performance. A demonstrator containing prototypes of the upgraded trigger electronic architecture has been installed on one of the barrel electromagnetic calorimeter readout front end crates to test it during the Run-2 campaign. The new architecture and its benefits for data taking will be discussed below as well as the results from first beam splash events.
\end{abstract}

Keywords: ATLAS, Liquid Argon, Calorimetry

\section{Electron and Photon Energy Calibration}

In the ATLAS [1] Liquid Argon (LAr) calorimeter, the calibration of the energy deposits of electrons, photons and converted photons consists of six different steps which are based either on simulation or on real data. First the reconstruction of electron and photon energies is optimized by a multivariate algorithm using single-particle simulation. In a second step, the response of the first two longitudinal layers is equalized in data with respect to simulation. The electron/photon response calibration from the first two steps is applied to cluster energies reconstructed from data and simulation samples in step three. Step four comprises a number of different uniformity correcจ tions such as the geometric intermodule widening, the time dependence of the high voltage in the presampler, or non-optimal high-voltage regions. The absolute energy scale in data is dea termined in a fifth step using a large number of $Z \rightarrow e e$ events which are also used in the same step to determine the resolution in data and smear the MC resolution to agree with that. In a sixth validation step, $J / \Psi \rightarrow e e, Z \rightarrow e e / Z \rightarrow l l \gamma$ events, binned in transverse energy are used to cross-check the calibration for electrons/photons. The final result is shown in Fig. 1.

\section{Trigger Upgrade and Demonstrator}

The LAr calorimeters have about 180000 readout cells of which the signals are digitized and then further processed in the back-end electronics. The instantaneous luminosities after the Phase-I upgrade [3] are expected to exceed the current peak luminosity $\left(8 \times 10^{33} \mathrm{~cm}^{-2} \mathrm{~s}^{-1}\right)$ by a factor four to nine. In order to

Email address: f spettel@mpp.mpg.de (Fabian Spettel)

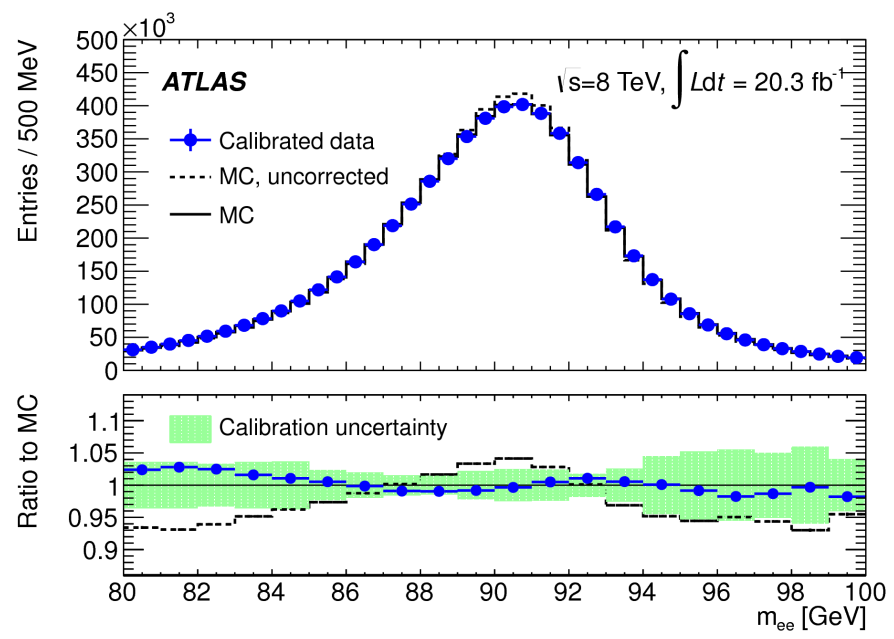

Figure 1: Top: Electron pair invariant mass distribution for $Z \rightarrow e e$ decays in data and improved simulation. Energy scale corrections are applied to the data. The improved simulation is shown before and after energy resolution corrections and is normalized to the number of events in data. Bottom: Ratio of the data and uncorrected MC distributions to the corrected MC distribution with the calibration uncertainty band [2].

maintain a manageable trigger output rate without rejecting too much low energy electron/photon events, a LAr trigger upgrade has been developed. This new architecture uses the concept of "Super-Cells" which provide information for each layer of the calorimeter and finer segmentation in pseudo-rapidity and azimuthal angle down to $\Delta \eta \times \Delta \phi=0.025 \times 0.1$ compared to the old granularity of the so called "Trigger Towers" which was $\Delta \eta \times \Delta \phi=0.1 \times 0.1$. The Super-Cells are illustrated in Fig. 2 . 


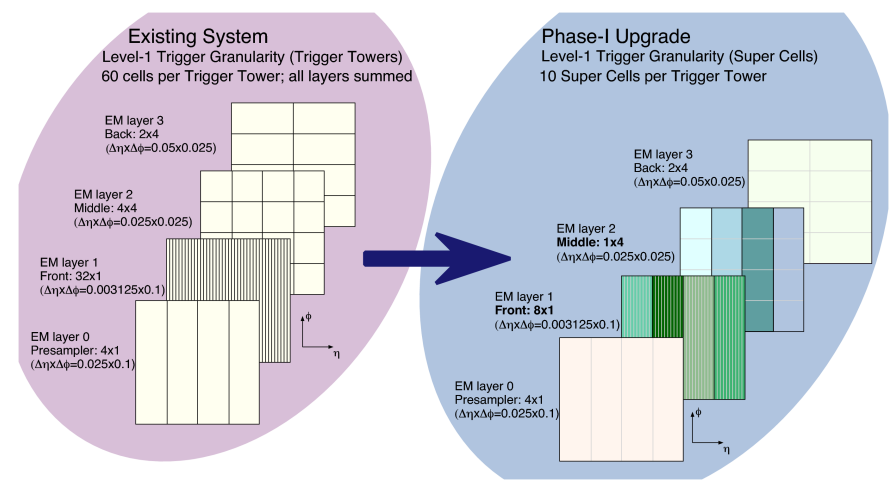

Figure 2: Existing Trigger Towers on the left (all of the indicated cells are summed to one tower) compared to Super-Cells with 10 times higher granularity on the right (the areas shown correspond to one Super-Cell for layer 0, four Super-Cells indicated by different colours in layer 1 and 2, respectively and one for layer 3) [3].

A schematic view of the Phase-I trigger architecture can be seen in Fig. 3. The first of the new components are the Layer Summing Boards which are mounted on the existing frontend boards and produce the finer granularity Super-Cell signals for each calorimeter layer. Additionally, there are new base planes in the front-end crates for analog signal transfer. The LAr Trigger Digitizer Boards (LTDBs) receive and digitize analog Super-Cell signals and further transmit the digitized ones to the backend, where the LAr Digital Processing System (LDPS) extracts the values of $E_{T}$ and transmits them to the level 1 calorimeter trigger system.

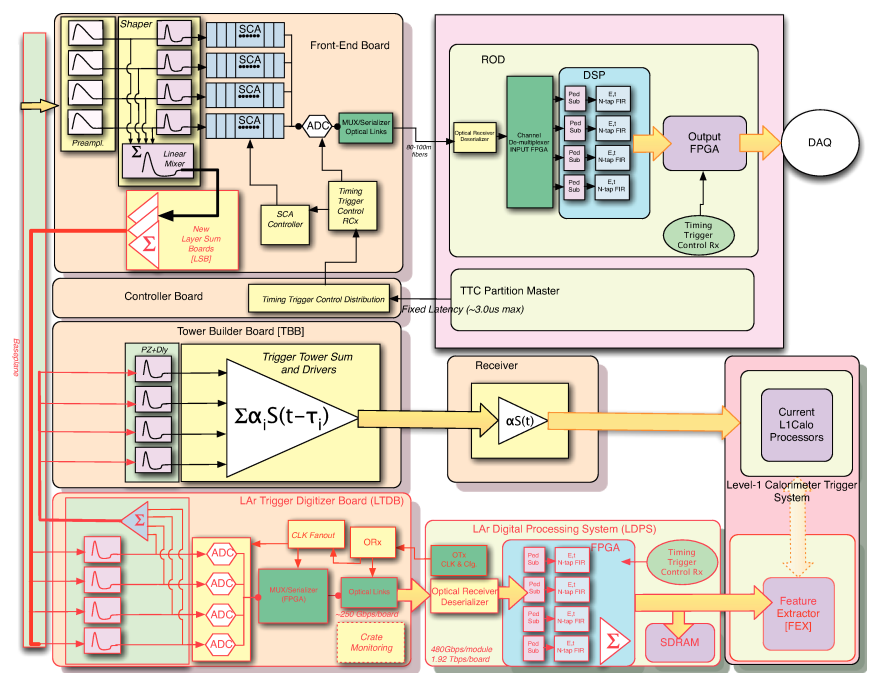

Figure 3: New trigger readout architecture, new components are indicated by the red outlines and arrows [3].

In order to test the upgrade electronics, a demonstrator system has been installed in the detector in 2014. It provides the full functionality of the new trigger architecture while maintaining the capability to build Trigger Towers to keep the old infrastructure operational during Run-2. Noise levels of the demonstrator have been tested by comparing it to the neighboring crate where one expects the same noise levels and amplitude shapes as in the demonstrator, see Fig. 4.

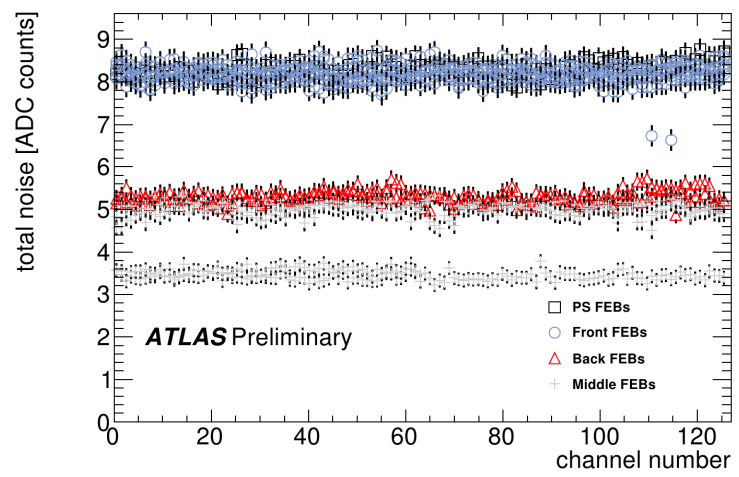

Figure 4: Cell noise for the Presampler, Front-, Middle-, and Back-layer in the current architecture. The noise levels shown here are identical to the noise levels of the neighoring module (not shown here) demonstrating that no additional noise is introduced by the new architecture [4].

\section{Beam Splash Events}

In April 2015, several beam splash events produced at the restart of the LHC were recorded allowing a quick scan of the calorimeter response at the beginning of Run-2. The proton beam arrives from one side and hits the collimator magnet which is located $175 \mathrm{~m}$ in front of the interaction point. The protons and fragmentation products subsequently shower and hit the detector from one side, causing every cell to light up. Fig. 5 shows the cell energy sums averaged over several splash events.
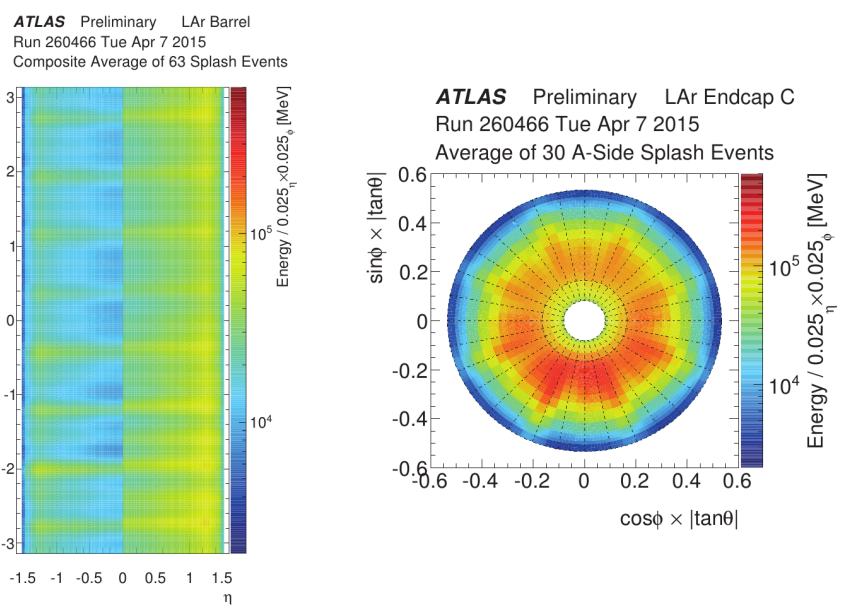

Figure 5: Average cell energy sums distributed in a $\Delta \eta \times \Delta \phi=0.025 \times 0.025$ grid. Left: 63 splash events from both LHC beams in the barrel calorimeter, 30 (33) of which were entering from the A (C) side and are plotted on the negative (positive) $\eta$-side. Right: The 30 events from the beam entering in the endcap $\mathrm{C}$ from the A side. A similar plot has been obtained for the 33 events entering in the endcap A from the $\mathrm{C}$ side [5].

\section{Conclusion}

In summary, the ATLAS LAr Calorimeters have shown excellent performance in Run-1 and are well prepared for the upcoming data taking periods.

\section{References}

[1] ATLAS Collab., JINST 3, S08003 (2008).

[2] ATLAS Collab., Eur. Phys. J. C74, 3071 (2014).

[3] ATLAS Collab., CERN-LHCC-2013-17, 2013.

[4] ATLAS Collab., https://twiki.cern.ch/twiki/bin/view/ AtlasPublic/LArCaloPublicResultsUpgrade, 2015.

[5] ATLAS Collab., https://twiki.cern.ch/twiki/bin/view/ AtlasPublic/LArCaloPublicResults2015, 2015. 